\title{
Les repas des Marocains
}

Comparaison entre Casablanca et des communes rurales $d u$ Souss (enquête de 2013)

\section{Moroccans' Meals}

\author{
A Comparative Study Between Casablanca and Souss Rural \\ Areas (A Survey Carried Out in 2013)
}

Laurence Tibère, Jean-Pierre Poulain, Nicolas Bricas, Driss Boumeggouti, Claude Fischler

Résumé : Cet article propose un panorama des habitudes des Marocains relatives aux repas, dans un contexte d'urbanisation et, plus largement, de mutations en cours dans les modes de vie et les aspirations sociales. L'analyse se fonde principalement sur les données d'une enquête quantitative menée entre 2012 et 2013. Elle porte sur les différences et les points de convergence entre les repas des Casablancais et ceux des habitants de communes rurales du Souss. Larticle pointe l'existence de situations différenciées entre les deux contextes, s'agissant en particulier du nombre des repas ou encore des sociabilités alimentaires. Il montre aussi la prégnance, en ville comme dans les villages ruraux, de certaines habitudes, telle que la valorisation de certains plats ou produits, ou l'importance de la commensalité.

Mots clés : Maroc, repas, rural, urbain

\begin{abstract}
This article provides an overview of Moroccan people's eating habits, in a context of urbanisation and, more broadly, of ongoing changes in lifestyles and social aspirations. The analysis is mainly based on data from a quantitative survey conducted between 2012 and 2013. It focuses on the differences and commonalities between the meals of Casablanca residents and those of the inhabitants of rural areas in the Souss region. The article points out the existence of differentiated
\end{abstract}


situations between the two contexts, particularly with regard to the number of meals or food sociability. It also shows the importance of certain habits, both in the city and in rural villages, such as the social valuation of certain dishes or products, or the importance of commensality.

Keywords: Morocco, meals, rural, urban

Du fait des mutations dans les modes de vie, les formes de sociabilité et les modèles sociaux (rapport au travail, structures familiales, parentalité, conjugalité...) qui l'accompagnent, l'urbanisation est un important facteur de changement dans l'alimentation, tant au niveau de la production et de la transformation que de la distribution et la consommation (Drenowski et Popkin 1997 ; Popkin 1999 ; Delisle 1990 ; 2012 ; Strigler, 2019). Plusieurs observateurs s'accordent sur le fait qu'au Maroc, où, en 2014, on comptait environ treize millions d'habitants en zone rurale (soit 40 pour cent de la population totale) pour vingt millions de citadins, des mutations sont visibles dans les habitudes alimentaires (HCP 2014 ; Banque mondiale 2020). Ces mutations sont liées à l'amélioration des conditions socio-économiques et de l'environnement alimentaire, mais aussi aux changements dans les modes de vie et les systèmes de valeurs (Sarno, Progano et al. 2003 ; Padilla 2008 ; Dernini et al. 2012 ; Allali 2017). Les conséquences habituellement associées à la transition alimentaire ont été repérées par différents auteurs, en particulier l'augmentation de la consommation de produits sucrés, d'aliments carnés et de matières grasses, et le recul des légumineuses. Les analyses convergent également sur le fait que, s'ils touchent les campagnes comme les villes, les changements ont une intensité différente dans les deux contextes. La disponibilité et le recours aux produits alimentaires prêts à la consommation, aux produits industriels (locaux ou importés) et la variété des produits consommés, seraient plus importants en ville (Delisle 2012 ; Erraoui 2016). Enfin, cette transition alimentaire sadosse aussi à l'apparition d'aspirations et de pratiques de consommation nouvelles dont plusieurs auteurs pointent la prégnance plus forte en milieu urbain (D’Addato 2006 ; Paterno et al. 2008; Sarter 2006 ; Zirari 2020).

Nous faisions l'hypothèse que l'existence de contrastes dans l'alimentation entre milieux urbain et rural n'excluait pas celle de certains points de convergence. D'abord parce qu'une grande partie des citadins est issue des zones rurales et que, même si une génération de néo-urbains née en ville émerge, les modèles issus des campagnes sont probablement encore actifs (Escallier 1990; Sarter 2006). Lautre point était que de nombreuses mobilités (d'individus et de produits) existent entre villes et campagnes, générant une perméabilité et des entrecroisements entre les deux univers (Fassin 1996 ; Pélissier 2000). On peut penser également que l'influence des medias, qu'il s'agisse des campagnes publicitaires ou encore d'informations nutritionnelles diffusées sur Internet, 
intervient dans les deux contextes, avec des effets sur l'alimentation. Selon l'Observatoire national du développement humain, la possession de la télévision s'est pratiquement généralisée, dans les villes comme dans les campagnes, souvent accompagnée par le récepteur satellite. Lélectrification du milieu rural et l'extension rapide de l'Internet via l'utilisation des smartphones jouent un rôle important également (ONDH 2012). Enfin, des phénomènes de patrimonialisation portés par les catégories sociales éduquées sont visibles en milieu urbain, à travers la réappropriation de pratiques perçues comme traditionnelles. Ces tendances ont été repérées dans d'autres régions du monde, notamment en Afrique ou en Amérique Latine, à travers la réhabilitation de certains plats considérés comme "nationaux " (Cusack 2000 ; Bricas 2008 ; MartinezLomeli 2020). Au Maroc, Poulain et Erraoui notent des dynamiques de revalorisation de certains plats tels que le couscous (Poulain 2006 ; Erraoui 2016).

Partant des repas, nous nous proposons d'explorer certaines mutations à l'œuvre dans l'alimentation et, plus largement, dans les modes de vie des Marocains. Larticle s'appuie sur des données collectées entre 2012 et 2013, auprès de 615 femmes et hommes âgés de 18 à 70 ans, vivant dans le Souss et dans la ville de Casablanca. Nous avons opté pour plusieurs communes rurales et une mégapole accueillant le cinquième des citadins du pays ${ }^{1}$. Ces données ne prétendent pas à la représentativité nationale, mais elles offrent une lecture en contraste entre espaces rural et urbain dans le Maroc contemporain. L'enquête mobilise une majorité de questions ouvertes sur les pratiques et les représentations relatives à l'alimentation. Nous avons été attentifs aux nombres et aux types de repas pris quotidiennement. Nous avons également examiné les contenus des repas, plus précisément, le statut de certaines composantes identifiées comme "traditionnelles » dans la littérature, et dont la consommation est en recul en milieu urbain au profit d'autres, plus récentes (Sarter 2006; Padilla 2008; Erraoui 2016). C'est le cas du tajine et du couscous, ou encore du pain fait maison, trois composantes emblématiques de la culture alimentaire marocaine, supports à la fois concrets et symboliques du lien au groupe (Tibère $2018 ; 2020$ ). Nous avons élargi l'analyse à celle de la sociabilité alimentaire, en ciblant les modes de préhension, les contextes de consommation, ainsi que l'organisation de la commensalité entre hommes et femmes. Sur ce dernier point, certains auteurs soulignent une plus grande mixité en ville qu'en zone rurale (Rachik 2005 ; Sarter 2006 ; Zirari 2020).

Avant d'aborder les résultats de l'enquête, nous présenterons la population étudiée ainsi que la méthodologie générale. Puis nous analyserons le nombre et les types de repas quotidiens, pour lesquels des différences sont effectivement visibles, notamment autour de la consommation du goûter. Nous poursuivrons par le contenu des repas, en ciblant notre analyse sur le pain et le tajine, avant d'aborder la commensalité et les formes de sociabilité alimentaire. Certains éléments relatifs aux représentations de nos interlocuteurs sur le «bien manger », ou encore sur leurs aspirations concernant l'alimentation, viendront compléter l’analyse. 


\section{Méthodologie et population étudiée}

Les données analysées sont issues d'une enquête plus large visant à saisir les habitudes alimentaires des Marocain-e-s, mais aussi certaines de leurs représentations en la matière ${ }^{2}$. La méthodologie a combiné une première phase qualitative exploratoire par entretiens individuels et discussions de groupe et une enquête par questionnaires ${ }^{3}$. Nous reprendrons quelques fois certains propos collectés lors de cette étape, en complément des données résultant de l'enquête quantitative. Dans celle-ci, nous nous sommes basés sur le rappel des dernières vingt-quatre heures pour accéder aux pratiques lors des repas ${ }^{4}$. Différents éléments de la prise alimentaire ont ainsi été répertoriés avec, pour les repas, une description de l'organisation interne du repas. Sont aussi précisés les lieux de consommation, la sociabilité, les modes de préhension et le fait de regarder ou non la télévision pendant le repas. L'enquête par questionnaire a été menée en face à face auprès de 305 personnes vivant à Casablanca et 310 dans le Souss, sur la base d'un questionnaire traduit en trois langues (français, arabe, berbère amazight). Les enquêteurs et enquêtrices étaient tous Marocains et formés aux spécificités de l'enquête. Les plans déchantillonnage ont été établis en collaboration avec les partenaires locaux et les services nationaux de statistiques (Haut-Commissariat au Plan et ministère de l'Economie et des Finances). Léchantillonnage a été réalisé par quotas, en milieu urbain comme rural, selon le sexe et lâge ${ }^{5}$. La population enquêtée compte 49,8 pour cent de femmes et 50,2 pour cent d'hommes, 38,3 pour cent des individus ont entre 18 et 35 ans, 56,3 pour cent, entre 36 et 65 ans et 5,4 pour cent sont âgés de plus de 65 ans. Ils sont majoritairement originaires du Souss (65,4 pour cent) par la naissance et, dans une moindre mesure, de zones urbaines autres que Casablanca $(24,2$ pour cent). Un indicateur d'insécurité alimentaire est mobilisé pour évaluer l'impact possible des conditions de vie sur le nombre de repas quotidiens ${ }^{6}$. Même si les liens avec lâge et le sexe sont évoqués, l'ambition principale de l'approche statistique était de mettre en évidence ce qui était corrélé avec le lieu de résidence (urbain ou rural) ${ }^{7}$.

\section{Résultats}

\section{Des différences autour du goûter}

Une différence souvent rapportée dans la littérature est l'existence de journées organisées autour de trois repas et d'une collation, plus fréquente dans les villages du Maroc, alors que le nombre de repas est moindre en ville (Sarter 2006 ; Padilla 2008 ; Allali 2017). Ces écarts sont attribués aux changements dans les rythmes sociaux et à la pression de l'activité professionnelle, plus forte dans les villes. Mais ils semblent également traduire des situations de précarité plus fréquentes dans les villes, où de plus fortes disparités socio-économiques 
sont repérables. On constate que, la veille de l’enquête, une majorité $(62,4$ pour cent) des individus des deux contextes a pris trois repas, plus précisément, un petit-déjeuner (ftour es-sbâh), un déjeuner (leghda), un diner (leacha), et une quatrième prise alimentaire appelée, selon les régions, "goûter ", «kaskrout ", "leachiya ", qui est en réalité un quatrième "petit repas", souvent composé de pain, et/ou de galettes de céréales, ou de crêpes, garnies de miel, de beurre, accompagné souvent de thé, parfois d'olives, et/ou d'une pâtisserie. Ces journées organisées autour de quatre repas, dont un goûter, sont effectivement plus fréquentes en milieu rural qu’à Casablanca. Ce que l'on peut dire des goûters, c'est qu'ils sont majoritairement consommés au domicile ( 80 pour cent), en particulier chez les femmes, à Casablanca. Ce résultat est peut-être associé aux observations récentes de Hayat Zirari qui, dans une enquête qualitative menée auprès de Casablancais en 2017, évoque le statut particulier du goûter au sein des familles des quartiers populaires. Elle précise que «ce moment de commensalité, partagé entre les enfants et les adultes, et parfois les visiteurs et voisins, est valorisé par les femmes de la classe moyenne (urbaine), parce que moins contraint que les repas " (Zirari 2020 : 37). Lauteure note que la prise d'un goûter permet souvent de ne prendre qu'un dîner léger, ou de ne pas dîner. Dans notre enquête, nous constatons également que les goûters pris à l'extérieur concernent majoritairement les hommes, à Casablanca comme en milieu rural. Les individus prenant trois repas quotidien, sans goûter, concernent un peu moins du tiers (27,5 pour cent) de la population globale, avec une prégnance plus forte chez les citadins. Enfin, c'est en ville que les journées ne comprenant que deux repas sont les plus fréquentes. Il s’agit majoritairement d'individus qui «sautent» le déjeuner (10,1 pour cent en tout). Le fait que les citadins en grave insécurité alimentaire soient surreprésentés pour ces situations pointe l'impact possible des situations de précarité en milieu urbain. Concernant l'activité professionnelle et l'influence des rythmes de travail, la surreprésentation des catégories " sans activité ", " employés » et "artisans " étant plus faible, nous la donnons à titre indicatif uniquement ${ }^{8}$.

\section{Le pain, toujours très présent}

Certains auteurs observent que, dans les villes marocaines, les aliments changent, mais ce sont surtout les façons de les consommer qui évoluent. Le blé y est présent sous forme de pain, de semoule, de pâtes, de pâtisseries, de pizzas, alors quà la campagne la gamme des produits dérivés est plus restreinte, tout comme pour le lait ou encore les oléagineux (Delisle 1990 ; Padilla 2008). Le pain est un aliment central de l'alimentation marocaine. Il accompagne le tajine au déjeuner ou au dîner, il est consommé au petit-déjeuner et au goûter. Sarter (2006) précise que, pendant longtemps, il a d’abord été consommé par les citadins, en particulier le pain blanc au levain, avant de devenir un aliment " noble », idéalisé dans les milieux populaires et dans les campagnes où il était mangé surtout noir, gris. Sarter rappelle que le pain a un caractère quasi sacré 
qu'exprime le mot neama (pain), souvent traduit par « don et grâce d'Allah ». La substitution récente des bouillies et des semoules de céréales par le pain dans les campagnes est liée notamment à l'introduction sur le marché de la farine panifiable manufacturée, dont la consommation a augmenté depuis les années soixante-dix. Sarter précise que, bien quau début du vingtième siècle l'usage du pain acheté sest développé en ville, le pain fait maison " reste entouré d'une aura et d'un prestige inégalés » (Sarter 2006 : 100). Il remarque toutefois, lors d'entretiens menés au milieu des années 2000, que la nouvelle génération n’a pas perpétué cette tradition du fait de sa pénibilité et du temps nécessaire, ayant ainsi recours au pain acheté, alors en voie de normalisation dans les villes. Lors de notre enquête, le pain fait maison a été consommé la veille par 77 pour cent des individus et le pain de boulangerie par 57,4 pour cent, avec une surreprésentation pour ce dernier à Casablanca. Le succès du second n'empêche pas l'usage du premier et met en évidence que les pratiques " traditionnelles ne sont pas radicalement incompatibles avec le changement, pas plus que la modernité avec la continuité » (Balandier 1971 : 107). Des formes de cohabitation entre anciennes et nouvelles habitudes sont ainsi fréquentes dans des contextes de transformation des sociétés (Tibère et Poulain 2019 ; Strigler 2019). Le recours au pain fait maison peut aussi traduire un récent retour aux méthodes culinaires traditionnelles que Erraoui (2016) attribue en partie à l'intégration des informations nutritionnelles diffusées dans les médias ou par le milieu médical. L'usage des huiles d’olive et d'argan, présentes à hauteur de 77 pour cent la veille de l'enquête, reflète probablement cette tendance. De plus, 39,7 pour cent des enquêtés déclarent recevoir des produits alimentaires de leur village d'origine au moins une fois par an, principalement des céréales, de l'huile d’olive ou d'argan, des fruits en coques, du miel, des produits laitiers, des fruits secs. Et la qualité gustative des aliments est perçue comme moins bonne en ville que dans les villages pour 66,2 pour cent des répondants, alors que la diversité alimentaire y est vue comme supérieure (79 pour cent). Nous avons mentionné la présence de pain au goûter : cet aliment est aussi consommé lors des autres repas. Les petits déjeuners, pris par 98 pour cent des individus la veille de l'enquête, sont composés très souvent ( 77 pour cent) de pain, parfois aussi de crêpes, accompagnés d'huile d'olive ou d'argan et/ou de beurre, de miel, parfois de produits laitiers (lait caillé, fromage Vache qui rit et Kiri, fromage gruyère) et souvent d'œufs. Une boisson chaude, en général du thé ${ }^{9}$ parfois du café au lait, est systématiquement consommée. Une différence s'affiche entre Casablanca et le Souss autour de la présence ou de l'absence de soupe ou bouillie de blé ou d'orge (dchicha, leassida, et belboula), souvent accompagnée de pain. Celle-ci est consommée par 14,5 pour cent des individus, avec une forte surreprésentation des ruraux. Lors des entretiens menés dans le Souss, cette catégorie de soupe était valorisée pour son statut nourrissant, en particulier lors de la rupture du jeûne du Ramadan. Reste quelle est aussi consommée dans certaines familles en dehors de cette période pour «bien commencer la journée », pour " tenir toute la matinée ». Le pain, 
omniprésent, est aussi consommé au cours des autres repas, en particulier avec le tajine.

\section{Qu'est ce qui est "tajiné »?}

Le terme tajine désigne à l'origine un récipient en argile, constitué d'un plat circulaire et d'un couvercle en forme de dôme. Toutefois, l'usage du mot pour désigner un ragoût, de légumes et/ou de viande, s’est généralisé. Selon Sarter (2006), les habitants du Moyen Atlas utilisent aussi le terme douaz pour désigner ce plat préparé d’ordinaire en faisant rissoler vivement de la viande découpée en gros morceaux dans de l'huile, accompagnée d’aromates, de persil, de coriandre, d'ail, d'oignons et d'épices, dont du poivre, du curcuma et du paprika. Sa cuisson, qui a lieu souvent dans des marmites ou en cocotteminute aujourd'hui, est menée à son terme en contrôlant le volume de liquide selon la quantité et la texture souhaitée pour la sauce. L'auteur note que le tajine est tellement associé au déjeuner au Maroc que le néologisme « tajiner » signifie préparer le déjeuner. Il rappelle qu'initialement le tajine est un plat d'origine urbaine qui sest généralisé à l’ensemble de la société marocaine, au point de devenir aujourd'hui l'un des plats emblématiques que les Marocains qualifient de "plat du Maroc », "spécialité numéro un », "plat national » (Rachick 2005; Sarter 2006 ; Padilla 2008). Qu'en est-il de sa consommation ? Certains plats emblématiques sont en effet valorisés dans les discours, sans forcément être consommés de façon régulière, en particulier dans les situations de changements rapides des sociétés (Tibère 2019 ; Tibère et Poulain 2019 ; Balandier 1971). Allali (2017) déclare que le tajine est encore très consommé au Maroc, surtout au déjeuner, parfois en plat unique, parfois accompagné de petites assiettes de salades ou de crudités. Sarter rappelle que sa consommation est indissociable de celle du pain, utilisé pour saucer tout en régulant la satiété du convive (Sarter 2006). Il souligne l'imaginaire social qui lui est associé, plat à la fois nourrissant, revigorant et, également, support du lien familial et social, modèle du "vrai repas » et du "bien manger». Nous avons constaté qu'à la question, "que signifie pour vous bien manger ? ", la satiété est mise en avant par 14,5 pour cent des individus ( «se remplir le ventre», " ne plus avoir faim», " avoir le ventre bien rempli »), juste après la santé (21 pour cent) qui apparaît dans les réponses du type " manger bon pour la santé », " manger équilibré », " manger des vitamines ». On repère également une valorisation déléments emblématiques (14,1 pour cent) parmi lesquels le tajine arrive largement en tête, suivi du couscous. Une différence intervient cependant entre ruraux et citadins pour cette question sur le "bien manger ", les premiers valorisant davantage la satiété et le tajine, et les seconds la santé. Ces résultats vont plutôt dans le sens d'une tendance à la "nutritionnalisation » de l'alimentation, plus marquée en milieu urbain, et d'un attachement, en zone rurale, au modèle nourrissant qu'incarnent certains plats comme le tajine (Poulain 2002; Deslile 2012). Ce dernier, aussi appelé « ragoût » par les citadins, est très présent dans 
les évocations des déjeuners de la veille (54 pour cent). Il est composé de pommes de terre et/ou légumes et toujours consommé avec du pain, parfois agrémenté d'une salade ou de poivrons et/ou d’olives en marinade. On trouve aussi des déjeuners de couscous (17,2 pour cent), composés souvent de viande et légumes, servis avec de la semoule de blé, et accompagnés la plupart du temps de lben (petit lait fermenté). Le couscous est symbole d'abondance et comporte un caractère social important puisqu'il est le plat des rassemblements familiaux et amicaux (Westermarck 2003 ; Sarter 2006). Cela explique en partie qu'il soit servi surtout le vendredi après la prière à la mosquée, voire, en milieux urbains, les week-ends, ce point étant selon Zirari (2020) un indice de sécularisation et/ou une adaptation aux contraintes de la vie urbaine casablancaise. En termes de consommation, le tajine et le couscous sont surreprésentés au déjeuner dans les villages du Souss.

\section{Moins de tajine au dîner}

Le repas du soir est traditionnellement considéré comme un repas secondaire au Maroc et il est fréquent qu'il soit moins consistant, plus simple que les autres (Sarter 2006). Dans notre enquête, 4,1 pour cent des individus ont un dîner composé de yaourt ou de quelques gâteaux avec du thé et 6 pour cent d'entre eux ne prennent rien, sans corrélation avec l'indicateur d'insécurité alimentaire. Certains repas sont composés de salades de légumes cuits ou de crudités (20,8 pour cent) et de pain, alors que les tajines,-sont un peu moins présents qu’au déjeuner (22,6 pour cent). Des pâtes (19,7 pour cent) sont également consommées, ce qui en soit n'est pas un fait nouveau au Maroc (Oubahli M. 2008). En revanche, l'usage des pâtes industrielles se généralise, et, déjà à la fin des années 2000, des enquêtes de consommation pointaient cette tendance liée la compression du temps culinaire et aux nouveaux goûts (Herradi 2008). Enfin, on trouve aussi des dîners composés de grillades, souvent accompagnées de fritures de légumes. Cette pratique est nettement surreprésentée en milieu urbain pour les deux repas (12 pour cent le midi, et 9,6 pour cent au dîner). Les grillades sont traditionnellement considérées comme moins nourrissantes et plutôt associées à des consommations secondaires ou aux déjeuners estivaux. Sarter (2006) note que les foyers au sein desquels elles se substituent au tajine en tant que plat principal sont plutôt composés de personnes jeunes et diplômées, souvent-aisées. Ces ménages mettent généralement en avant leurs qualités nutritionnelles et diététiques, mais aussi une certaine lassitude face au tajine. Ce succès est dû aussi au fait que les grillades sont faciles à préparer, voire, dans certains cas, sont achetées déjà préparées. Ces pratiques, de même que les livraisons de repas au domicile en milieu urbain, notamment de tacos ou pizzas, connaissent un certain succès aujourd'hui auprès des jeunes générations et des femmes désireuses de sortir de la routine, mais aussi de moins cuisiner $^{10}$. Si la consommation de grillades n'est pas un fait nouveau au Maroc, 
son statut de plat principal, sous forme de steaks, de kefta ou encore de brochettes, l'est (Boumeggouti 2013).

\section{Une commensalité très active}

Le partage de nourriture est un marqueur important de l'identité culturelle marocaine, en particulier autour du repas. Selon Sarter, la commensalité est liée à la baraka, notion qui tient une place importante au Maroc. Il rappelle la proposition de Colin de traduire le terme par «force bénéfique d’origine divine qui provoque la surabondance dans le domaine physique, la prospérité et la félicité dans l'ordre psychique » (Colin 1965 : 1063 ; Sarter 2006 : 78). Sarter relate les propos d'une Marocaine ayant vécu en France : " quand on mange dans un plat commun, on est plus facilement rassasié que quand on mange chacun dans son assiette comme en France. C'est parce que quand tu manges seul ta portion paraît plus petite que quand tu manges en commun ». Il observe aussi que les mères de familles sont réticentes à scinder leur préparation en plusieurs assiettes, affirmant que si elles divisent le tajine, celui-ci perd la baraka (Sarter $2006: 80$ ). Des travaux d'anthropologie religieuse et politique montrent que, traditionnellement, la baraka est aussi conçue comme une force qui agit pour le maintien ou le renforcement de la cohésion sociale (Jamous 1981 ; Geertz 1986 ; Maunier 1998). Le repas pris en commun est vu comme une mise en scène de la solidarité familiale et de la cohésion du groupe, en dépit du développement de l'individualisme et du recul de la séparation traditionnelle entre les hommes et les femmes (Sarter 2006) ${ }^{11}$. A la fin des années 2000, Padilla attribuait la baisse de la consommation de produits et de plats traditionnels dans la plupart des groupes sociaux non pas à un attachement moindre à leur égard, excepté chez les plus jeunes, mais davantage à la diminution des occasions de les consommer (Padilla 2008). Parmi les citadins de notre enquête, dont la majorité est originaire du Souss, on perçoit une nostalgie des repas partagés puisque 74,8 pour cent des répondants estiment quon mange plus souvent seul en ville et que 61,5 pour cent le déplorent. Pourtant, l'analyse des modalités de consommation de la veille montre que la commensalité est relativement forte, en zone rurale et en milieu urbain. On observe en effet que les repas partagés représentent 77,3 pour cent pour les goûters, 82 pour cent pour les déjeuners, 96 pour cent des dîners et moins pour les petits déjeuners ( 34,5 pour cent), ces derniers étant réglés sur des horaires différenciés de départ du domicile. On peut souligner par ailleurs que femmes et hommes mangent ensemble dans 60,8 pour cent des cas au déjeuner, 72,5 pour cent au dîner, 50 pour cent au petit-déjeuner et 51,5 pour cent pour le goûter, ces résultats pointant les changements à l'œuvre sur ce plan. Les discours en faveur de la commensalité entre hommes et femmes vont en partie dans ce sens pour 59,5 pour cent des répondants des deux contextes. Concernant les modes de préhension, les repas consommés au sein du foyer 
dans le plat commun (à la main où à la cuiller) concernent 62 pour cent des individus pour le déjeuner et 76,8 pour cent pour le dîner, sans lien significatif avec le lieu de résidence. L'usage de l'assiette individuelle est plus fort en milieu urbain, en particulier pour le dîner. Enfin, 57,1 pour cent des enquêtés déclarent manger devant la télévision pour le déjeuner et 65,7 pour cent pour le dîner, sans différence entre contextes rural et urbain. Qu'en est-il de l'alimentation hors foyer ? Padilla déclare qu'en zone urbaine « les opportunités de consommation familiale régressent avec les modes de vie urbains qui poussent à la restauration hors du domicile »(Padilla 2008 : 156). Lauteure précise que la prolifération des lieux traditionnels de consommation, tels que les mahlabas ${ }^{12}$, et une forte consommation de raieb (le lait caillé) comme snack, en particulier chez les jeunes, en sont les indicateurs. Les repas pris à l'extérieur concernent, dans l'enquête, 20 pour cent des individus, dont un peu plus de la moitié dans le cadre professionnel. Deux tendances sont repérables, qu'il s'agisse de l'univers professionnel ou de loisirs : d'une part, la surreprésentation de la classe d'âge 26-35 ans et, d'autre part, celle des hommes. La différenciation de genre autour des sociabilités alimentaires extérieures était assez marquée à l'époque de l'enquête, mais il est possible que des changements interviennent sur ce plan depuis, comme l'indique l'analyse de Zirari sur les aspirations des femmes, en particulier dans les villes. En 2017, l'auteure observe ainsi des transformations significatives dans les représentations des femmes vis-à-vis de leur statut, leur et rôle social, et la montée d'aspirations individuelles au mieux-être et à l'épanouissement (Zirari 2020). La préparation culinaire et les modes de consommation alimentaire sont l'un des espaces dans lequel se déploient ces dynamiques. Selon l'auteure, la pluralité des styles de vie et des usages sociaux qui caractérise l'espace urbain favorise l'émergence de nouveaux comportements, comme le fait de "manger une nourriture préparée ailleurs que dans la cuisine familiale, comme dans les mahlabas, ou achetée auprès de vendeurs de snacks ambulant " (Zirari 2020 : 36). Zirari observe lémergence, en fin de semaine, de nouvelles formes de sociabilité alimentaire à Casablanca. Selon elle, ces pratiques permettent aux femmes qui ont un emploi de se décharger du travail culinaire. Ces phénomènes évoluent lentement cependant et nous avions relevé chez les principales intéressées un certain attachement à ce rôle social. En effet, si les réponses à l'affirmation « les hommes n’ont pas à entrer dans la cuisine " reflétaient plutôt des aspirations au changement, pour 59,3 pour cent des individus ("pas d'accord»), sans différence selon le lieu de l'enquête, nous constations que les femmes étaient surreprésentées parmi les enquêtés qui choisissaient la modalité " d'accord ». Pour conclure sur les repas hors foyer, nous constatons qu'ils sont aussi l'objet d'une assez forte commensalité au déjeuner (67 pour cent) et au repas du soir ( 77,5 pour cent), un peu moins au goûter ( 56,2 pour cent) et au petit-déjeuner ( 43,4 pour cent). La différenciation entre milieu urbain et rural réside dans le fait que les consommations du dehors, extraprofessionnelles, sont surreprésentées à Casablanca, plus précisément pour le petit-déjeuner et le goûter. Et, à côté du " café » et 
des « restaurants », qu'ils fréquentent tout comme les répondants du Souss, les Casablancais goûtent aussi " dans la rue », " au marché », "sur le chemin » (11 pour cent). Les modalités de consommation hors foyer apparaissent ainsi plus diversifiées en zone urbaine mais, aussi, plus nomades.

\section{Conclusion}

Lobjectif de cet article était de dresser un panorama des changements dans les habitudes alimentaires des Marocains. La comparaison entre urbain et rural et l'entrée par les repas ont permis de saisir les différences et les permanences des contextes familiaux et sociaux à Casablanca, ainsi que dans les zones rurales du Souss. Les données recueillies montrent un nombre des repas quotidiens plus faible en ville. Cette différence est liée au niveau social et aux rythmes de vie urbains, mais aussi aux situations de précarité plus fréquentes en contexte urbain. Des écarts sont visibles également s'agissant des modes de consommation et de la sociabilité alimentaire, plus diversifiée et plus ouverte sur l'extérieur en ville. Laccès et le recours à des formes d'approvisionnement comme les superettes et les supermarchés favorise la consommation de certains produits associés à la modernité, tel que le pain acheté à la boulangerie, ou encore les pâtes alimentaires industrielles. Toutefois, les changements qui accompagnent des transformations des modes de vie et des systèmes de valeurs cohabitent avec des consommations et des rituels qui dessinent une spécificité culturelle marocaine. Le tajine, par exemple, très présent dans les déjeuners, en ville comme à la campagne, en fait partie, de même que la commensalité, et les manières de manger, toujours actives dans les deux contextes, ou encore la préparation du pain à la maison qui cohabite avec son achat en boulangerie. En contexte urbain, des réaménagements s'opèrent dans les pratiques et les représentations relatives au repas sous diverses influences, celles d'un environnement plus ouvert, en termes d'offre, de rythme de vie et de consommation, de types de populations, de messages médiatiques, mais aussi celle de milieux ruraux avec lesquels les interactions sont à la fois idéelles et réelles. Sept années se sont écoulées depuis cette étude et l'on constate que dans les villes, à Casablanca mais aussi à Rabat ou Marrakech, certaines tendances s'installent. La sociabilité alimentaire urbaine se diversifie encore davantage, avec la montée de nouvelles formes de distribution alimentaire et de restauration hors foyer. A côté de l'essor des restaurants de fast-food, la livraison de nourritures au domicile connait un vrai succès, facilitée par la présence d’applications numériques dédiées. En ville comme à la campagne, l’accès à l'Internet et l'usage des smartphones se généralisent, accentuant les échanges au sein du pays et avec l'extérieur. Enfin, la valorisation d'un " équilibre alimentaire » combinant plaisir et santé s’accentue, en particulier sur les réseaux sociaux, à travers les discussions autour de la nutrition, et le fréquent partage de vidéos, d'articles, démissions, ou encore de conférences sur ce thème. 
Laurence Tibère est maître de conférences (hab.) en sociologie à l'Université de Toulouse Jean Jaurès (France), et membre du CERTOP (Centre de recherche sur le travail, l'organisation, le pouvoir), UMR-CNRS 5044. Ses recherches portent sur les dimensions sociales et culturelles de l'alimentation, en lien avec des questions de lien social mais aussi de santé publique. Elle enseigne à l'ISTHIA où elle est en charge d'un Master "sciences sociales appliquées à l'alimentation ». Elle est membre de la Chaire d'études alimentaires, cultures et santé à l'Université Taylors (Malaisie) où elle est enseignante et chercheuse. Email: tibere@univ-tlse2.fr

Laurence Tibère is an associate professor in sociology at the University of Toulouse, Jean Jaures (France), and a member of CERTOP (Research Centre d'Etude et de Recherche Travail, Organisation, Pouvoir), UMR-CNRS 5044. She has conducted researches on the links between food and Culture, not only related to social ties but also to health. She teaches at ISTHIA (Toulouse School of Tourism, Hospitality Management and Food Studies) where she is in charge of a Master's Degree Program in Food Studies. She is a member of the Chair of Food Studies Cultures and Health at Taylors University (Malaysia) where she is a lecturer and a researcher. Email: tibere@univ-tlse2.fr

Jean-Pierre Poulain est professeur de sociologie à l'université Jean Jaurès de Toulouse. Ses travaux portent sur la «sociologie de l'alimentation» et les «études alimentaires». Il mène des recherches sur les liens entre alimentation, cultures et santé dans le cadre du CERTOP UMR-CNRS 5044, et dirige, depuis 2012, la chaire «Food Studies» mise en place conjointement par l'université de Toulouse (France) et l'université Taylor (Kuala Lumpur, Malaisie). Il est le co-directeur du Laboratoire international associé (LIA-CNRS) «Alimentation, cultures et santé» Email: poulain@univ-tlse2.fr

Jean-Pierre Poulain is a professor of sociology at Toulouse University, Jean Jaurès, focusing on "sociology of food" and "food studies". He conducts researches on the links between food, cultures and health in the frame of CERTOP UMR-CNRS 5044, and has led, since 2012, the chair of "Food Studies" conjointly set up by the University of Toulouse (France) and the Taylor's University (Kuala Lumpur, Malaysia). He is the co-director of the International Associated Laboratory (LIA-CNRS) "Food, Cultures, and Health”. Email: poulain@univ-tlse2.fr

Driss Boumeggouti est maître de conférences en géographie à l'université de Toulouse Jean Jaurès. Ses recherches portent sur le Maroc, le tourisme, la géopolitique et le développement. Il est responsable d'un Master en tourisme (ISTHIA).Email: driss.boumeggouti@univ-tlse2.fr 
Driss Boumeggouti is a associate professor in geography at Toulouse University, Jean Jaurès. His research focuses on tourism, geopolitics, and development in Morocco. He's in charge of a Master's Degree Program in Tourism (ISTHIA). Email: driss.boumeggouti@univ-tlse2.fr

Nicolas Bricas est Socio-économiste, directeur de recherche au CIRADMontpellier (UMR MOISA). Il est co-responsable du Mastère spécialisé "Innovations et politiques pour une alimentation durable" - MS IPAD de Montpellier SupAgro et du CIRAD. Son expertise porte sur la sécurité alimentaire et l'alimentation durable, les comportements alimentaires et les systèmes alimentaires urbains. Il est membre du Groupe international d'experts sur les systèmes alimentaires durables (IPES-Aliments). Il dirige la Chaire UNESCO Alimentations du monde.Email: nicolas.bricas@cirad.fr

Nicolas Bricas is a socio-economist, research director at CIRAD-Montpellier. $\mathrm{He}$ is co-responsible for the Specialized Master's Degree Program in "Innovations and Policies for Sustainable Food"-MS IPAD of Montpellier SupAgro and CIRAD. His expertise relates to food security and sustainable food, eating behaviors, and urban food systems. He is a member of the International Panel of Experts on Sustainable Food Systems (IPES-Food), and is Director of the UNESCO Chair in World Food Systems. Email: nicolas.bricas@cirad.fr

Claude Fischler est sociologue, directeur de recherche émérite au CNRS. Il dirige l'Institut interdisciplinaire d'anthropologie contemporaine, unité de recherche de l'EHESS, à Paris. Email: claude.fischler@ehess.fr

Claude Fischler is a sociologist and an emeritus research director at CNRS. He chairs the Interdisciplinary Institute of Contemporary Anthropology, a research unit of EHESS, in Paris. Email: claude.fischler@ehess.fr

\section{Notes}

1. Ces derniers résident principalement dans sept grands centres urbains : le Grand Casablanca, Fes, Tanger, Marrakech, Salé, Meknès et Rabat (HCP 2014).

2. Il s'agit du programme ALIMI-ANR-08-ALIA-003, intitulé « Food Culture and Migrants: Consequences for Food Policies », sous la direction de Claude Fischler du Centre Edgard Morin / IIAC-CNRS-EHESS. Partenaires : UMR Moisa/CIRAD, Nicolas Bricas (dir.), UMR Nutripass/IRD, Bernard Maire (dir.) et UMR CERTOP/ CNRS-5044, Jean-Pierre Poulain (dir.). Ont aussi participé : Driss Boumeggouti, Natacha Calandre, Mila Lebrun, Evelyne Ribert, Emilie Terranova, Marie Etien, Daniela Chiang, Michelle Holdworth, Nadia Hibbi et Laurence Tibère.

3. Vingt-six entretiens approfondis ont été réalisés dans le Souss et dix-sept à Casablanca. 
4. La collecte s'est déroulée sur toute la semaine.

5. Six communes rurales ont été retenues dans la région du Souss : il s’agit de Massa, Tighmi, Aglou, Idaousmlal, Maader, Azni. S’agissant du milieu urbain, deux arrondissements de Casablanca ont été enquêtés : Ben M'Sik et Moulay Rachid.

6. L'indicateur de sécurité alimentaire a été construit selon la méthode dite "FANTA » (Food and Nutrition Technical Assistance) (Coates et al. 2007). Il indique que 52,9 pour cent des individus sont en situation de sécurité alimentaire à Casablanca, 52 pour cent dans le Souss, 8,4 pour cent et 14,3 pour cent en insécurité légère, 21,9 pour cent contre 19 pour cent en insécurité modérée, et 16,8 pour cent et 14,6 pour cent en grande insécurité.

7. Lanalyse statistique a été réalisée sur la base du test Khi2 (de Pearson) à l'aide du logiciel SPSS (VS 14.0).

8. Il s'agit plus particulièrement de garagistes et de menuisiers à leur compte, ainsi que de coiffeurs/coiffeuses, chauffeurs.

9. Le thé est d'ailleurs présent tout au long de la journée, lors—et en dehors-des repas des enquêtés.

10. Selon les observations menées par Driss Boumeggouti à Casablanca et Agadir entre 2016 et 2020.

11. L'auteur parle "d'euphémisation des inégalités » lors des repas et rappelle que, traditionnellement, l'accès de chacun à l'alimentation durant le repas est réglé selon sa position et son statut social, tels que les définit la société patriarcale : les hommes adultes sont privilégiés par rapport aux femmes et aux enfants, les aînés ont la préséance au sein de la fratrie, les garçons l'emportent sur leurs sœurs (Sarter $2006: 83$ ).

12. Il s'agit de snacks qui préparent et vendent principalement des produits laitiers. Ce type de structure a eu un grand succès dans les années quatre-vingt à l'échelle du pays, en partant des grandes villes. Elles se sont diversifiées en termes d'offre et de services (restauration rapide).

\section{Bibliographie}

Allali F. (2017), 'Evolution des pratiques alimentaires au Maroc', International Journal of Medicine and Surgery 4, Special Issue, ID 145 : 70-73.

Balandier G. (1971), Sens et puissance (Paris : Quadrige, Presses universitaires de France).

Boumeggouti D. (2013), 'La Kéfta du Gharb au Maroc, un savoir-faire en voie de disparition', Horizons Maghrébins, no. 69, Manger au Maghreb: Le goût de la viande, partie III : 118-133.

Bricas N. (2006), 'La pluralité des références identitaires des styles alimentaires urbains en Afrique', communication au GDR Economie \& Sociologie « Les Marchés Agroalimentaires», 22-23 Mars, 149-159.

Cardon, P. (2018), 'La commensalité familiale sous tension', in Quand manger fait société, (ed.) P. Cardon, (Lille : Presses universitaires du Septentrion).

Coates J., Swindale A. et Bilinsky P. (2007), Echelle de l'Accès déterminant l'Insécurité alimentaire des Ménages (EAIAM) pour la mesure de l'accès alimentaire des ménages: Guide d'indicateurs (version 3), Washington, D.C., Projet d'Assistance technique en 
matière d'Alimentation et de Nutrition (FANTA), Académie pour le Développement de l'Education.

Colin, G.S. (1965), 'Baraka, Encyclopédie de l'Islam vol. II, 1063. (Paris : G. P. Maisonneuve et Larose).

Corbeau J.-P. (2002), 'Itinéraires de mangeurs', Penser l'alimentation : Entre imaginaire et rationalité, (dir.) J.-P.Poulain, (Toulouse, Editions Privat).

Cusack, I. (2000), 'African Cuisine: Recipes for Nation-Building', Journal of African Studies 13, no. 2: 207-225.

D'Addato, A. V. (2006), 'Progression to Third Birth in Morocco in the Context of Fertility Transition', Demographic Research 15, no. 19: 517-536.

Delisle H. (1990), Urban Food Consumption Patterns in Developing Countries-Some Issues and Challenges, rapport réalisé pour la FAO.

Delisle H. (2012), 'Transition nutritionnelle', in Dictionnaire des cultures alimentaires, (dir) Poulain J.-P., (Paris : Presses universitaires de France), 1449-1453.

Dernini, S., Berry, E., Bach-Faig, A., Belahsen, R., Donini, L., Lairon, D., SerraMajem, L. et Cannella, C. (2012), 'Un modèle alimentaire construit par les scientifiques', in MediTERRA, La diète méditerranéenne pour un développement régional durable, (ed.) CIHEAM (Paris : Presses de Sciences Po), 73-91.

Douglas, M. (1984), Food in the Social Order (New York: Russell Sage Foundation).

Drewnowski, A. et Popkin, B.M. (1997), 'The Nutrition Transition : New Trends in the Global Diet', Nutrition Reviews 55, no. 2 : 31-43.

El Ansari, R. (2009), 'Dynamique régionale et développement inégal au Maroc', communication au colloque international Inégalités et développement dans les pays méditerranéens, Université de Galatasaray, Istanbul, Turquie, 21-23 mai 2009.

Enquête panel de ménages 2012 : Rapport des premiers résultats, Observatoire national du développement humain, Royaume du Maroc, Rabat.

Erraoui, E.H. (2016), 'Le système alimentaire marocain, entre mondialisation et pratiques territorialisées', communication au colloque MEDCOP CLIMAT, Tanger, 12 juillet 2016.

Escallier, R. (1990), 'Urbanisation et développement au Maroc', Cahiers de la Méditerranée 41, no. 1, États et pouvoirs en Méditerranée (XVIe-XXe siècles) : Mélanges offerts à André Nouschi, tome I : 163-182.

Fassin D., (1996), 'L'expérience des villes', Enquête [En ligne], 4, mis en ligne le 11 juillet 2013, http://journals.openedition.org/enquete/73, 10.4000/ enquete.733.

Geertz, C. (1986), Savoir local, savoir global (Paris : Presse universitaire de France).

Herpin, N. (1988), 'Le repas comme institution : Compte rendu d'une enquête exploratoire', Revue française de sociologie 29, no. 3, Lécole en croissance : 503-521.

Herradi, J.E. (2008), 'L'industrie des pâtes alimentaires, le Maroc loin derrière la Tunisie et l'Algérie', L'Economiste, 15 septembre.

Jamous, R. (1981), Honneur et baraka (Paris : Maison des sciences de l'Homme).

Martinez-Lomeli, L. (2020), “ "Manger dehors » dans les villes de Mexico et de Guadalajara : de quelques tensions entre les dimensions sanitaires et patrimoniales au Mexique, in Manger en ville: Regards socio-anthropologiques d'Afrique, d'Amérique latine et d'Asie, (dir.) A. Soula, C. Yount-André, O. Lepiller, N. Bricas, (Versailles : Editions Quae), 57-69.

Maunier, R. (1998), Recherches sur les échanges rituels en Afrique du Nord (Paris : Bouchene). 
Oubahli, M. (2008), 'Une histoire de pâte en Méditerranée occidentale : Des pâtes arabo-berbères et de leur diffusion en Europe latine au Moyen Âge (Partie II). La France et le monde italique', Horizons Maghrébins, no. 59, Manger au Maghreb, partie II : 14-29.

Padilla, M. (2008), 'Alimentation et évolution de la consommation', in MediTERRA, Les futurs agricoles et alimentaires en Méditerranée, (ed.) CIHEAM, (Paris : Presses de Sciences Po), 149-167.

Paterno, A., Gabrielli, G., D’Addato, A. V. (2008), 'Travail des femmes, caractéristiques familiales et sociales : le cas du Maroc', Cahiers québécois de démographie 37, no. 2 : 263-289, https://doi.org/10.7202/038133ar.

Pélissier, P. (2000), 'Les interactions rurales/urbaines en Afrique de l'Ouest et Centrale', Bulletin de l'APAD [En ligne], no. 19, mis en ligne le 12 juillet 2006, http://journals .openedition.org/apad/422.

Popkin B.M. (1999), 'Urbanization, Lifestyle Changes and the Nutrition Transition: World Development', 27, no. 11 : 1905-1916.

Poulain, J.-P. (2001), Manger en France aujourd'hui : Attitudes, normes et pratiques (Toulouse : Privat).

Poulain, J.-P. (2006), 'Les modèles alimentaires méditerranéens : un héritage pluriel à étudier pour en faire un label pour le futur', Horizons Maghrébins, no. 55 : 8-28.

Poulain, J.-P. (2002), Sociologies de l'alimentation: Le mangeur et l'espace social alimentaire (Paris : Presses universitaires de France).

Rachik, H. (2005), Rapport de synthèse de l'enquête nationale sur les valeurs: Cinquantenaire de l'indépendance du Maroc. Cinquante ans de développement humain et Perspectives 2025, Rabat.

Rapport sur l'évolution de l'urbanisation au Maroc, Données de la Banque mondiale, Perspective Monde 2020.

Résultats de l'enquête nationale sur la consommation et les dépenses des ménages 20132014, Haut Commissariat au Plan du royaume du Maroc, Rabat, 2016.

Sarter, G. (2006), 'Manger et élever des moutons au Maroc : Sociologie des préférences et des pratiques de consommation et de production de viande' (Thèse de doctorat en sociologie sous la direction de Maxime Aubert, L'Université Paris I, IEDES).

Strigler, F. (2019), Mutations alimentaires au Laos: Salade de Papaye ou pizza? (Paris : Editions Karthala).

Tibère, L. (2018), 'La construction sociale de «l'en commun » par la consommation : les sociétés réunionnaise et malaisienne', Revue Hommes et migrations, no. 1320 : 31-39.

Tibère, L. (2021, à paraître), Nourritures en commun : La construction sociale de la relation à l'Autre (Paris : Editions des Archives contemporaines).

Tibère, L., Poulain, J-P. (2019), 'La modernité alimentaire dans les territoires français d'Outre-mer', Cahiers de Nutrition et Diététique (Paris : Elveisier).

Zirari, H. (2020), '(S'en) sortir de la cuisine ! Reconfigurations des rapports de genre et pratiques alimentaires à Casablanca', in Manger en ville : Regards socio-anthropologiques d'Afrique, d'Amérique latine et d'Asie, (dir.) A. Soula, C. Yount-André, O. Lepiller, N. Bricas (Versailles : Editions Quae), 33-45.

Westermarck, E. (2003), Les cérémonies du mariage au Maroc (Paris : Editions du Jasmin). 